\title{
COMPACT BILINEAR OPERATORS AND COMMUTATORS
}

\author{
ÁRPÁD BÉNYI AND RODOLFO H. TORRES \\ (Communicated by Michael T. Lacey)
}

\begin{abstract}
A notion of compactness in the bilinear setting is explored. Moreover, commutators of bilinear Calderón-Zygmund operators and multiplication by functions in a certain subspace of the space of functions of bounded mean oscillations are shown to be compact.
\end{abstract}

\section{INTRODUCTION}

What is a compact bilinear operator, and what is a good non-trivial example of it? This article originates from these simple and natural questions. Natural, because we understand rather completely the notion of continuity (or boundedness) of bilinear operators, and the notions of continuity and compactness are tightly connected in the linear setting.

Interestingly, the notion of compactness in the multilinear setting can be traced back to the foundational article of Calderón [3. The natural definition given in [3. p. 119] can easily be seen to be equivalent to the one we will present. But, to the best of our knowledge, the notion of compactness in this setting has only been considered within the context of interpolation; see also the recent work by Fernandez and da Silva [6] for more on the relation between interpolation and compact multilinear operators.

Naturally appearing concrete examples of compact bilinear operators seem to be absent in the literature until now. However, the experience in several different contexts tells that commutators operators with special symbols tend to be "better" than just bounded. In fact, a second motivation for this article is to extend to the bilinear setting a theorem of Uchiyama 18, about linear commutators of Calderón-Zygmund operators and pointwise multiplication. Uchiyama improved the boundedness result of Coifman, Rochberg and Weiss 4 to compactness when the symbol is in an appropriate subspace of the John-Nirenberg space $B M O$.

In order to draw an analogy between the notions of continuity and compactness in the bilinear setting, we briefly recall the two basic notions of separate and joint continuity for a bilinear operator. Therefore let $X, Y, Z$ be normed spaces, and let $\|\cdot\|_{X},\|\cdot\|_{Y},\|\cdot\|_{Z}$ denote their corresponding norms; we will drop the corresponding indices if the space in question is clear from the context. A bilinear operator $T: X \times Y \rightarrow Z$ is defined through its section operators: $T_{x}=T(x, \cdot): Y \rightarrow Z$ and

Received by the editors January 5, 2012.

2010 Mathematics Subject Classification. Primary 42B20, 47B07; Secondary 42B25, 47G99.

Key words and phrases. Bilinear operators, compact operators, singular integrals, CalderónZygmund theory, commutators.

The second author was supported in part by the National Science Foundation grant DMS 1069015. 
$T_{y}=T(\cdot, y): X \rightarrow Z$ are required to be linear for all (fixed) $x \in X, y \in Y$. The two definitions we have in mind are the following:

(1) (Separate continuity) $T$ is separately continuous if $T_{x}$ and $T_{y}$ are continuous for all $x \in X, y \in Y$.

(2) (Joint continuity) $T$ is (jointly) continuous if it is continuous from $X \times Y$ to $Z$; the product space is normed, say, via $\|(x, y)\|=\|x\|+\|y\|, x \in X$, $y \in Y$.

As usual, the norm of the bilinear operator $T$ is defined to be

$$
\|T\|=\inf \{M>0:\|T(x, y)\| \leq M\|x\|\|y\|, \text { for all }(x, y) \in X \times Y\},
$$

and we say that $T$ is bounded if $\|T\|<\infty$. It is a well-known simple exercise to show that the notions of continuity and boundedness are equivalent. Also trivially, continuity implies separate continuity. Moreover, somewhat surprisingly, the two notions are equivalent if either of the spaces $X$ or $Y$ is assumed to be Banach. This known fact is proved in Rudin's book [13, Theorem 2.17] under the more general assumption that $X$ is an $F$-space and $Y$ is metrizable. The proof relies on the Banach-Steinhaus principle. Under the stronger hypothesis that both $X$ and $Y$ are Banach, it is stated as an exercise in Schechter's book [15, Exercise 24, p. 74]. It is worth pointing out that the completeness of either one of the spaces $X$ or $Y$ is crucial. For example, it is straightforward to check that if we let $X=Y=Z=$ $C(0,1)$, the space of continuous functions on $[0,1]$, and we endow it with the $L^{1}$ norm (hence, making $X$ incomplete), then $T(f, g)=f \cdot g$ is separately continuous but not continuous.

Taking into consideration the brief discussion above, we are naturally led to consider the two notions of (joint) compactness and separate compactness for a bilinear operator. The next section is devoted to the definitions and basic properties of such operators, many of which are proved in the Appendix. The extension to the bilinear setting of the result of Uchiyama mentioned before is presented in Section 3.

\section{Compact AND SEPARATEly COMPACT BILINEAR OPERATORS}

In what follows, we write $B_{r, X}=\{x \in X:\|x\| \leq r\}$ to denote the closed ball of radius $r$ centered at the origin in the normed space $X$. Again, when the context is clear, we will drop the index $X$ and simply write $B_{r}$.

Definition 1. A bilinear operator $T: X \times Y \rightarrow Z$ is called (jointly) compact if $T\left(B_{1, X} \times B_{1, Y}\right)$ is precompact in $Z$.

Proposition 1. Let $T: X \times Y \rightarrow Z$ be a bilinear operator. Then the following statements are equivalent:

(c1) $T$ is compact.

(c2) $T\left(B_{1}, X \times Y\right)$ is precompact.

(c3) For all $r>0, T\left(B_{r, X \times Y}\right)$ is precompact.

(c4) For all $r_{1}, r_{2}>0, T\left(B_{r_{1}, X} \times B_{r_{2}, Y}\right)$ is precompact.

(c5) For all bounded $B \subset X \times Y, T(B)$ is precompact.

(c6) For all bounded $B^{1} \subset X, B^{2} \subset Y, T\left(B^{1} \times B^{2}\right)$ is precompact.

(c7) For all bounded sequences $\left\{\left(x_{n}, y_{n}\right)\right\} \subset X \times Y$, the sequence $\left\{T\left(x_{n}, y_{n}\right)\right\}$

has a convergent subsequence.

Furthermore, if $Z$ is Banach, then (c1) is also equivalent to:

(c8) For all bounded $B \subset X \times Y, T(B)$ is totally bounded. 
The collection of all bounded bilinear operators $T: X \times Y \rightarrow Z$ is denoted by $\mathcal{B}(X \times Y, Z)$. The collection of all compact bilinear operators $T: X \times Y \rightarrow Z$ is denoted by $\mathcal{K}(X \times Y, Z)$. With this notation, we have the following.

Proposition 2. If $T_{1}, T_{2} \in \mathcal{K}(X \times Y, Z)$ and $\alpha, \beta \in \mathbb{C}$, then $T=\alpha T_{1}+\beta T_{2} \in$ $\mathcal{K}(X \times Y, Z)$.

The proofs of Propositions 1 and 2 are straightforward, and we include them for the convenience of the reader in the Appendix. In particular, (c8) is how compact bilinear operators are defined in 3 .

An immediate consequence of Proposition 2 is given next.

Corollary 1. $\mathcal{K}(X \times Y, Z)$ is a linear subspace of $\mathcal{B}(X \times Y, Z)$.

Proof. The fact that $\mathcal{K}(X \times Y, Z)$ is a vector space follows directly from Proposition 2 For the inclusion $\mathcal{K}(X \times Y, Z) \subset \mathcal{B}(X \times Y, Z)$, assume that the compact bilinear operator $T$ is not bounded. Then, for some $r>0$, there exists a sequence $\left(x_{n}, y_{n}\right) \in$ $B_{r, X \times Y}$, and $\left\|T\left(x_{n}, y_{n}\right)\right\| \rightarrow \infty$. Thus, $\left\{T\left(x_{n}, y_{n}\right)\right\}$ could not have a convergent subsequence, so $T\left(B_{r, X \times Y}\right)$ would not be precompact, contradicting (c3).

The statement of the following result, which will be used in the next section, can also be found in [3]. For completeness, we provide a succinct proof.

Proposition 3. If $Z$ is Banach, then $\mathcal{K}(X \times Y, Z)$ is closed in $\mathcal{B}(X \times Y, Z)$.

Proof. Let $T_{n} \in \mathcal{K}(X \times Y, Z)$ and $T \in \mathcal{B}(X \times Y, Z)$ be such that $T_{n} \rightarrow T$. We want to show that $T \in \mathcal{K}(X \times Y, Z)$. To this end, let $\left\{\left(x_{n}, y_{n}\right)\right\}$ be a bounded sequence in $X \times Y$. Since $T_{1}$ is compact, there exists a subsequence $\left\{\left(x_{n_{k}^{1}}, y_{n_{k}^{1}}\right)\right\}$ such that $\left\{T_{1}\left(x_{n_{k}^{1}}, y_{n_{k}^{1}}\right)\right\}$ is convergent. Since $T_{2}$ is compact, we can extract $\left\{\left(x_{n_{k}^{2}}, y_{n_{k}^{2}}\right)\right\}$ as a subsequence of $\left\{\left(x_{n_{k}^{1}}, y_{n_{k}^{1}}\right)\right\}$ such that $\left\{T_{2}\left(x_{n_{k}^{2}}, y_{n_{k}^{2}}\right)\right\}$ is convergent. If we let $x_{j}=x_{n_{j}^{j}}, y_{j}=y_{n_{j}^{j}}$, then $\left\{T_{n}\left(x_{j}, y_{j}\right)\right\}_{j \geq 1}$ is convergent for all $n \geq 1$. We have

$\left\|T\left(x_{i}, y_{i}\right)-T\left(x_{j}, y_{j}\right)\right\| \leq\left\|T-T_{n}\right\|\left(\left\|x_{i}\right\|\left\|y_{i}\right\|+\left\|x_{j}\right\|\left\|y_{j}\right\|\right)+\left\|T_{n}\left(x_{i}, y_{i}\right)-T_{n}\left(x_{j}, y_{j}\right)\right\|$.

Since $\left(x_{j}\right)$ and $\left(y_{j}\right)$ are bounded sequences, for some $M>0$ we have $\left\|x_{j}\right\|\left\|y_{j}\right\| \leq M$ for all $j \geq 1$. Let $\epsilon>0$. Because $T_{n} \rightarrow T$, there exists some $N \in \mathbb{N}$ such that for all $n \geq N,\left\|T-T_{n}\right\|<\epsilon /(4 M)$. Since $\left\{T_{N}\left(x_{j}, y_{j}\right)\right\}_{j \geq 1}$ is Cauchy, there exists a $J \in \mathbb{N}$ such that for all $i, j \geq J,\left\|T_{N}\left(x_{i}, y_{i}\right)-T_{N}\left(x_{j}, y_{j}\right)\right\|<\epsilon / 2$. Thus, $\| T\left(x_{i}, y_{i}\right)-$ $T\left(x_{j}, y_{j}\right) \|<\epsilon$ for all $i, j \geq J$, which proves that $\left\{T\left(x_{i}, y_{i}\right)\right\}$ is Cauchy and, since $Z$ is Banach, also convergent. By (c7), we get that $T$ must be compact.

As in the linear case, compact bilinear operators interact nicely with bounded ones via composition. Since this property plays no role in Section [3, we refer the interested reader to the Appendix for a brief discussion of this topic. After this introduction to the notion of compactness, we seek out the notion of separate compactness.

Definition 2. A bilinear operator $T: X \times Y \rightarrow Z$ is called compact in the first variable if $T_{y}: X \rightarrow Z$ is compact for all $y \in Y$. $T$ is called compact in the second variable if $T_{x}: Y \rightarrow Z$ is compact for all $x \in Y$. $T$ is called separately compact if $T$ is compact in both the first and second variables. 
As expected, the immediate observation now is that the notion of separate compactness is weaker than that of compactness. Indeed, for a fixed $x \in X$, let $r>0$ be such that $\|x\|<r$, that is, $x \in B_{r, X}$. Then

$$
\overline{T_{x}\left(B_{1, Y}\right)} \subset \overline{T\left(B_{1+r, X \times Y}\right)},
$$

and then use the fact that a closed subset of a compact set is necessarily compact; see Proposition 1. A similar reasoning applies to $T_{y}$.

As above, we also see that a separately compact bilinear operator $T$ must be separately continuous. But recall that this does not necessarily mean $T$ is continuous; in fact, we will show an example of a separately compact operator which is not bounded. Nevertheless, the assumption that $T$ is bounded could be added in both Definitions 1 and 2 as long as at least one of the spaces $X$ or $Y$ is Banach. A few elementary examples illustrating the two notions of compactness are detailed in the Appendix. However, our main interest in the notion of compactness stems from some important objects in harmonic analysis: the bilinear commutators.

\section{Bilinear COMmutators}

Let $T$ be a bilinear Calderón-Zygmund operator as defined in [7] and assume (for simplicity) that the kernels $K$ and $\nabla K$ satisfy the usual conditions in such a theory. Let $b, b_{1}, b_{2} \in B M O\left(\mathbb{R}^{n}\right)$. We are interested in the following three bilinear commutators:

$$
\begin{aligned}
{[T, b]_{1}(f, g)(x) } & =(T(b f, g)-b T(f, g))(x), \\
{[T, b]_{2}(f, g)(x) } & =(T(f, b g)-b T(f, g))(x), \\
{\left[\left[T, b_{1}\right]_{1}, b_{2}\right]_{2}(f, g)(x) } & =\left(\left[T, b_{1}\right]_{1}\left(f, b_{2} g\right)-b_{2}\left[T, b_{1}\right]_{1}(f, g)\right)(x) .
\end{aligned}
$$

Formally, they take the form

$$
\begin{aligned}
{[T, b]_{1}(f, g)(x) } & =\int_{\mathbb{R}^{n}} \int_{\mathbb{R}^{n}} K(x, y, z)(b(y)-b(x)) f(y) g(z) d y d z, \\
{[T, b]_{2}(f, g)(x) } & =\int_{\mathbb{R}^{n}} \int_{\mathbb{R}^{n}} K(x, y, z)(b(z)-b(x)) f(y) g(z) d y d z, \\
{\left[\left[T, b_{1}\right]_{1}, b_{2}\right]_{2}(f, g)(x) } & =\int_{\mathbb{R}^{n}} \int_{\mathbb{R}^{n}} K(x, y, z)\left(b_{1}(y)-b_{1}(x)\right)\left(b_{2}(z)-b_{2}(x)\right) f(y) g(z) d y d z .
\end{aligned}
$$

By the results in [12, [17, 9], and [11, these operators map $L^{p} \times L^{q} \rightarrow L^{r}$ with $1 / p+1 / q=1 / r$ for all $1<p, q<\infty$, with estimates of the form

$$
\begin{gathered}
\left\|[T, b]_{1}(f, g)\right\|_{L^{r}},\left\|[T, b]_{2}(f, g)\right\|_{L^{r}} \lesssim\|b\|_{B M O}\|f\|_{L^{p}}\|g\|_{L^{q}}, \\
\left\|\left[\left[T, b_{1}\right]_{1}, b_{2}\right]_{2}(f, g)\right\|_{L^{r}} \lesssim\left\|b_{1}\right\|_{B M O}\left\|b_{2}\right\|_{B M O}\|f\|_{L^{p}}\|g\|_{L^{q}} .
\end{gathered}
$$

We will show their compactness properties when the symbols $b, b_{1}, b_{2}$ are in $C M O$. In this article, $C M O$ denotes the closure of $C_{c}^{\infty}$ in the $B M O$ topology. As pointed out by Bourdaud et al. in 2, this space coincides with VMO (the space of functions of vanishing mean oscillation) as studied by Coifman and Weiss in [5], but differs from other versions of $V M O$ found in the literature. In particular, the original $V M O$ space of Sarason [14 is the closure of the uniformly continuous functions in $B M O$, which coincides with $C M O$ in the the case of the torus but not in $\mathbb{R}^{n}$. The notation $C M O$ (also used by Uchiyama) seems to go back to Neri [10]; see the historical comments in Bourdaud [1. See also Stein's book [16, Chapter IV] for 
further related properties of $B M O$. We will only need the fact that, by definition then, $C_{c}^{\infty}$ is dense in $C M O$.

The results we shall prove should be viewed as the bilinear counterparts of Theorem 2 of Uchiyama 18. As in [18, we will rely on the Fréchet-Kolmogorov Theorem characterizing the precompactness of a set in $L^{r}$. More precisely (see Yosida's book [19, p. 275]), a set $\mathcal{H}$ is precompact in $L^{r}, 1 \leq r<\infty$, if and only if

$$
\sup _{h \in \mathcal{H}}\|h\|_{L^{r}}<\infty
$$

$$
\lim _{A \rightarrow \infty}\|h\|_{L^{r}(\{|x|>A\}}=0 \quad \text { uniformly in } h \in \mathcal{H},
$$

and

$$
\lim _{t \rightarrow 0}\|h(\cdot+t)-h(\cdot)\|_{L^{r}}=0 \quad \text { uniformly in } h \in \mathcal{H} .
$$

Theorem 1. If $b \in C M O, 1 / p+1 / q=1 / r, 1<p, q<\infty$ and $1 \leq r<\infty$, then $[T, b]_{1}: L^{p} \times L^{q} \rightarrow L^{r}$ is compact. Similarly, if $b_{1}, b_{2}$ are also in $C M O$, then $\left[T, b_{2}\right]_{2}$ and $\left.\left[T, b_{1}\right]_{1}, b_{2}\right]_{2}$ are compact for the same range of exponents.

Proof. By (1) and Proposition 3 it is enough to show the result for $b \in C_{c}^{\infty}$. Moreover, given the boundedness of the operator and a density argument, to apply (3) - (5) in our situation it will be enough to prove that for all $f, g \in C_{c}^{\infty}$ the following two conditions hold:

a) Given $\epsilon>0$, there exists an $A>0(A=A(\epsilon)$ but independent of $f$ and $g)$ with the property that

$$
\left(\int_{|x|>A}\left|[T, b]_{1}(f, g)(x)\right|^{r} d x\right)^{1 / r} \lesssim \epsilon\|f\|_{L^{p}}\|g\|_{L^{q}} .
$$

b) Given $\epsilon \in(0,1)$ there exists a sufficiently small $t_{0}\left(t_{0}=t_{0}(\epsilon)\right.$ but independent of $f$ and $g$ ) such that for all $0<|t|<t_{0}$,

$$
\left\|[T, b]_{1}(f, g)(\cdot)-[T, b]_{1}(f, g)(\cdot+t)\right\|_{L^{r}} \lesssim \epsilon\|f\|_{L^{p}}\|g\|_{L^{q}} .
$$

We pick $A>1$ sufficiently large so that $|x|>A$ implies $x \notin \operatorname{supp} b$; in particular, we will pick $A>2 \max \{|y|: y \in \operatorname{supp} b\}$. We have the following sequence of inequalities:

$$
\begin{aligned}
\left|[T, b]_{1}(f, g)(x)\right| & \leq \iint_{y \in \operatorname{supp} b}|K(x, y, z)||b(y)||f(y) \| g(z)| d y d z \\
& \leq\|b\|_{L^{\infty}} \iint_{y \in \operatorname{supp} b} \frac{|f(y)||g(z)|}{(|x-y|+|x-z|)^{2 n}} d y d z \\
& \leq \int_{y \in \operatorname{supp} b} \frac{|f(y)|}{|x-y|^{n}} \int \frac{|g(z)|}{(|x-y|+|x-z|)^{n}} d z d y \\
& \leq 2^{n}|x|^{-n} \int_{y \in \operatorname{supp} b}|f(y)|\left(\int(|x-y|+|x-z|)^{-n q^{\prime}} d z\right)^{1 / q^{\prime}} d y\left\|_{g}\right\|_{L^{q}} \\
& \leq 2^{n}|x|^{-n}|\operatorname{supp} b|^{1 / p^{\prime}}\|f\|_{L^{p}}\left(\int\left(1 / 2+|z|^{-n q^{\prime}}\right) d z\right)^{1 / q^{\prime}}\|g\|_{L^{q}} \\
& \lesssim|x|^{-n}|\operatorname{supp} b|^{1 / p^{\prime}}\|f\|_{L^{p}}\|g\|_{L^{q}} .
\end{aligned}
$$

The previous inequality now allows us to integrate $\left|[T, b]_{1}(f, g)(x)\right|^{r}$ over the set $\{x:|x|>A\}$ and to obtain (6). 
To prove (7) we first decompose the expression inside the $L^{r}$ norm on the left hand side as follows:

$$
[T, b]_{1}(f, g)(x)-[T, b]_{1}(f, g)(x+t)=A(x)+B(x)+C(x)+D(x),
$$

where, for a convenient choice of $\delta>0$ to be specified later,

$$
\begin{aligned}
& A(x)=\iint_{|x-y|+|x-z|>\delta} K(x, y, z)(b(x+t)-b(x)) f(y) g(z) d y d z, \\
& B(x)=\iint_{|x-y|+|x-z|>\delta}(K(x, y, z)-K(x+t, y, z))(b(y)-b(x+t)) f(y) g(z) d y d z, \\
& C(x)=\iint_{|x-y|+|x-z|<\delta} K(x, y, z)(b(y)-b(x)) f(y) g(z) d y d z, \\
& D(x)=\iint_{|x-y|+|x-z|<\delta} K(x+t, y, z)(b(x+t)-b(y)) f(y) g(z) d y d z .
\end{aligned}
$$

If we now let $T_{*}(f, g)$ denote the maximal truncated bilinear singular integral operator

$$
T_{*}(f, g)(x)=\sup _{\delta>0}\left|\iint_{|x-y|+|x-z|>\delta} K(x, y, z) f(y) g(z) d y d z\right|,
$$

then

$$
|A(x)| \leq|b(x+t)-b(x)|\left|T_{*}(f, g)(x)\right| .
$$

By a result of Grafakos-Torres [8] (see the arguments on p. 1264 therein), we obtain

$$
\|A\|_{L^{r}} \leq|t|\|\nabla b\|_{L^{\infty}}\|f\|_{L^{p}}\|g\|_{L^{q}} .
$$

In order to estimate $B(x)$, we use the smoothness estimate on the kernel $K$ and get

$$
\begin{aligned}
|B(x)| \lesssim|t||| b \|_{L^{\infty}} & \left(\int_{|x-y|>\delta / 2} \int \frac{|f(y)||g(z)|}{(|x-y|+|x-z|)^{2 n+1}} d y d z\right. \\
& \left.+\int_{|x-z|>\delta / 2} \int \frac{|f(y)||g(z)|}{(|x-y|+|x-z|)^{2 n+1}} d y d z\right),
\end{aligned}
$$

provided $|t|<\delta / 4$. The two terms inside the parentheses are symmetric in $y, z$; thus we estimate them the same way. For example, we have

$\int_{|x-z|>\delta / 2} \int \frac{|f(y)||g(z)|}{(|x-y|+|x-z|)^{2 n+1}} d y d z=\int_{|x-z|>\delta / 2} \frac{|g(z)|}{|x-z|^{n+1}}\left(\phi_{|x-z|} *|f|\right)(x) d z$, where we denote by $\phi_{s}(x)=s^{-n} \phi\left(s^{-1} x\right)$, with $\phi(x)=(|x|+1)^{-2 n-1}$, an integrable, radial, decreasing function. We can bound pointwise each of the terms above as follows:

$$
\left(\phi_{|x-z|} *|f|\right)(x) \leq M(|f|)(x) \text { and } \int_{|x-z|>\delta / 2} \frac{|g(z)|}{|x-z|^{n+1}} \lesssim \frac{1}{\delta} M(|g|)(x),
$$

where $M(f)(x)$ denotes the Hardy-Littlewood maximal function. Therefore, we conclude that

$$
|B(x)| \lesssim \frac{|t|}{\delta}\|b\|_{L^{\infty}} M(|f|)(x) M(|g|)(x) .
$$

The $L^{p}$ boundedness of the maximal operator then gives

$$
\|B\|_{L^{r}} \lesssim \frac{|t|}{\delta}\|b\|_{L^{\infty}}\|f\|_{L^{p}}\|g\|_{L^{q}}
$$


To estimate the third term, we use the size estimate of the Calderón-Zygmund kernel $K$. We have

$$
\begin{aligned}
|C(x)| & \leq\|\nabla b\|_{L^{\infty}} \iint_{|x-y|+|x-z|<\delta} \frac{|x-y|}{(|x-y|+|x-z|)^{2 n}}|f(y) \| g(z)| d y d z \\
& \leq\|\nabla b\|_{L^{\infty}} \iint_{|x-y|+|x-z|<\delta} \frac{|f(y)||g(z)|}{(|x-y|+|x-z|)^{2 n-1}} d y d z \\
& \leq\|\nabla b\|_{L^{\infty} \delta}\left(\delta^{-1 / 2} \int_{|x-y|<\delta} \frac{|f(y)|}{|x-y|^{n-1 / 2}} d y\right)\left(\delta^{-1 / 2} \int_{|x-z|<\delta} \frac{|g(z)|}{|x-z|^{n-1 / 2}} d z\right) \\
& \lesssim \delta\|\nabla b\|_{L^{\infty}} M(|f|)(x) M(|g|)(x) .
\end{aligned}
$$

Hence,

$$
\|C\|_{L^{r}} \lesssim \delta\|\nabla b\|_{L^{\infty}}\|f\|_{L^{p}}\|g\|_{L^{q}} .
$$

Finally, for the last term we proceed in an analogous manner by replacing $x$ with $x+t$ and the region of integration $\{y:|x-y|<\delta\}$ with the larger one $\{y$ : $|(x+t)-y|<\delta+|t|\}$. Thus

$$
\begin{aligned}
&|D(x)| \leq\|\nabla b\|_{L^{\infty}}(\delta+|t|)^{1 / 2} \delta^{1 / 2}(\left.(\delta+|t|)^{-1 / 2} \int_{|(x+t)-y|<\delta+|t|} \frac{|f(y)|}{|(x+t)-y|^{n-1 / 2}} d y\right) \\
& \times\left(\delta^{-1 / 2} \int_{|x-z|<\delta} \frac{|g(z)|}{|x-z|^{n-1 / 2}} d z\right) \\
& \leq(\delta+|t|)^{1 / 2} \delta^{1 / 2}\|\nabla b\|_{L^{\infty}} M(|f|)(x+t) M(|g|)(x) .
\end{aligned}
$$

This now leads to

$$
\|D\|_{L^{r}} \lesssim \delta^{1 / 2}(\delta+|t|)^{1 / 2}\|\nabla b\|_{L^{\infty}}\|f\|_{L^{p}}\|g\|_{L^{q}} .
$$

Let us now define $t_{0}=\frac{\epsilon^{2}}{8\left(1+\|b\|_{L} \infty+\|\nabla b\|_{L} \infty\right)}$, and for each $0<|t|<t_{0}$, select $\delta=|t| / \epsilon$. Inequalities (8)-(11) imply (7). Combining this with the inequalities (1) and (6), we conclude that $[T, b]_{1}$ is compact. In a completely analogous way, if $b_{2} \in C M O$, then $\left[T, b_{2}\right]_{2}$ is compact.

Moreover, although for general symbols in $B M O$ the second order commutator is harder to study, for symbols in $C M O$ it turns out to be easy to handle because of the extra cancellation, so we only sketch the arguments needed. As before, we may assume that $b_{1}, b_{2} \in C_{c}^{\infty}$, and we need to check two conditions, a) and b), similar to the ones before.

The first condition that we need is in fact easier to check compared to the argument we gave for (6). Let us select a sufficiently large $A$ such that $A>2 \max \{|x|$ : $\left.x \in \operatorname{supp} b_{1} \cup \operatorname{supp} b_{2}\right\}$, and so that for $|x|>A$ we have $x \notin \operatorname{supp} b_{1} \cap \operatorname{supp} b_{2}$. The size estimate on the kernel $K$ yields the following sequence of inequalities:

$$
\begin{aligned}
\left|\left[\left[T, b_{1}\right]_{1}, b_{2}\right]_{2}(f, g)(x)\right| & \leq\left\|b_{1}\right\|_{L^{\infty}}\left\|b_{2}\right\|_{L^{\infty}} \int_{y \in \operatorname{supp} b_{1}} \int_{z \in \operatorname{supp} b_{2}} \frac{|f(y)||g(z)| d y d z}{(|x-y|+|x-z|)^{2 n}} \\
& \leq\left\|b_{1}\right\|_{L^{\infty}}\left\|b_{2}\right\|_{L^{\infty}} \int_{y \in \operatorname{supp} b_{1}} \frac{|f(y)|}{|x-y|^{n}} d y \int_{z \in \operatorname{supp} b_{2}} \frac{|g(z)|}{|x-z|^{n}} d z \\
& \lesssim\left\|b_{1}\right\|_{L^{\infty}}\left\|b_{2}\right\|_{L^{\infty}}\|f\|_{L^{p}}\|g\|_{L^{q}}\left|\operatorname{supp} b_{1}\right|^{1 / p^{\prime}}\left|\operatorname{supp} b_{2}\right|^{1 / q^{\prime}}|x|^{-2 n}
\end{aligned}
$$


The transition to the last inequality used the fact that if $|x|>A$, then $\min (\mid x-$ $y|| x-z \mid,) \geq|x| / 2$. In particular, we easily obtain

$$
\left(\int_{|x|>A}\left|\left[\left[T, b_{1}\right]_{1}, b_{2}\right]_{2}(f, g)(x)\right|^{r} d x\right)^{1 / r} \lesssim \epsilon\|f\|_{L^{p}}\|g\|_{L^{q}} .
$$

Our final task is to prove an $L^{r}$ estimate on

$$
\left[\left[T, b_{1}\right]_{1}, b_{2}\right]_{2}(f, g)(x)-\left[\left[T, b_{1}\right]_{1}, b_{2}\right]_{2}(f, g)(x+t) \text {. }
$$

In analogy to the decomposition done for (7), we break this into a sum of four terms,

$$
E(x)+F(x)+G(x)+H(x)
$$

where

$$
\begin{aligned}
E(x) & =\iint_{|x-y|+|x-z|>\delta} K(x, y, z)\left(b_{1}(x+t)-b_{1}(x)\right)\left(b_{2}(z)-b_{2}(x)\right) f(y) g(z) d y d z, \\
F(x) & =\iint_{|x-y|+|x-z|>\delta}\left(K(x, y, z)\left(b_{2}(z)-b_{2}(x)\right)-K(x+t, y, z)\left(b_{2}(z)-b_{2}(x+t)\right)\right) \\
& \times\left(b_{1}(y)-b_{1}(x+t)\right) f(y) g(z) d y d z, \\
H(x) & =\iint_{|x-y|+|x-z|<\delta} K(x, y, z)\left(b_{1}(y)-b_{1}(x)\right)\left(b_{2}(z)-b_{2}(x)\right) f(y) g(z) d y d z, \\
H(x)=\iint_{|x-y|+|x-z|<\delta} K(x+t, y, z)\left(b_{1}(y)-b_{1}(x+t)\right) & \times\left(b_{2}(x+t)-b_{2}(z)\right) f(y) g(z) d y d z .
\end{aligned}
$$

The terms $E, G, H$ are estimated, with slight changes, using the same tools as in the proof for $[T, b]_{1}$. For example, if we consider the $E$ term, we can write, similarly to the $A$ term before,

$$
|E(x)| \leq\left|b_{1}(x+t)-b_{1}(x)\right|\left(\left|T_{*}\left(f, b_{2} g\right)(x)\right|+\left|b_{2}(x)\right|\left|T_{*}(f, g)(x)\right|\right) .
$$

Thus, we get

$$
\begin{aligned}
\|E\|_{L^{r}} & \leq|t|\left\|\nabla b_{1}\right\|_{L^{\infty}}\left(\|f\|_{L^{p}}\left\|b_{2} g\right\|_{L^{q}}+\left\|b_{2}\right\|_{L^{\infty}}\|f\|_{L^{p}}\|g\|_{L^{q}}\right) \\
& \leq 2|t|\left\|\nabla b_{1}\right\|_{L^{\infty}}\left\|b_{2}\right\|_{L^{\infty}}\|f\|_{L^{p}}\|g\|_{L^{q}} .
\end{aligned}
$$

To deal with the $F$ term, we slightly rearrange the term inside the integral and write $F$ as a sum of two terms, $F_{1}+F_{2}$, where

$$
\begin{aligned}
F_{1}(x)=\iint & (K(x, y, z)-K(x+t, y, z))\left(b_{1}(y)-b_{1}(x+t)\right) \\
& \times\left(b_{2}(z)-b_{2}(x+t)\right) f(y) g(z) d y d z \\
F_{2}(x)=\iint & \int(x, y, z)\left(b_{1}(y)-b_{1}(x+t)\right)\left(b_{1}(x+t)-b_{1}(x)\right) f(y) g(z) d y d z ;
\end{aligned}
$$

the integration is, of course, over the set $\{(y, z):|x-y|+|x-z|>\delta\}$.

Now, the $F_{1}$ term corresponds to having two $B$ terms as before:

$$
\begin{aligned}
& \iint(K(x, y, z)-K(x+t, y, z))\left(b_{1}(y)-b_{1}(x+t)\right) f(y)\left(b_{2} g\right)(z) d y d z \text { and } \\
& b_{2}(x+t) \iint(K(x, y, z)-K(x+t, y, z))\left(b_{1}(y)-b_{1}(x)\right) f(y) g(z) d y d z
\end{aligned}
$$


Thus, the only difference in their estimate, compared to what we have done for the $B$ term, is an extra multiplying $\left\|b_{2}\right\|_{L^{\infty}}$ term. This, of course, has no effect on the outcome.

For the $F_{2}$ term we do something similar, except that this behaves more like two $A$ terms as before. First, we factor out the $b_{1}(x+t)-b_{1}(x)$, which will contribute a multiple $t\left\|\nabla b_{1}\right\|_{L^{\infty}}$. The remaining part is

$$
\iint K(x, y, z)\left(b_{1} f\right)(y) g(z) d y d z-b_{1}(x+t) \iint K(x, y, z) f(y) g(z) d y d z,
$$

which we estimate as the term $E$ above. The $b_{1}$ is now absorbed in the maximal function $T_{*}$ by the function $f$, while in $E$ it was absorbed by $g$.

Combining all the estimates for the terms $E, F, G, H$, we end up with the desired control

$$
\left\|\left[\left[T, b_{1}\right]_{1}, b_{2}\right]_{2}(f, g)(x)-\left[\left[T, b_{1}\right]_{1}, b_{2}\right]_{2}(f, g)(x+t)\right\|_{L^{r}} \lesssim \epsilon\|f\|_{L^{p}}\|g\|_{L^{q}},
$$

when $|t|$ is chosen sufficiently small depending on $\epsilon$. Estimates (2), (12) and (13) guarantee, via (3)-(5), the compactness of the second order commutator.

\section{Appendix}

4.1. Proof of Proposition 1) ( 1 1) $\Rightarrow(\mathrm{c} 2)$ Recall that we endowed $X \times Y$ with the norm $\|(x, y)\|=\|x\|+\|y\|$. It is clear that

$$
B_{1, X \times Y} \subset B_{1, X} \times B_{1, Y} \subset B_{2, X \times Y}=2 B_{1, X \times Y},
$$

and therefore

$$
\overline{T\left(B_{1, X \times Y}\right)} \subset \overline{T\left(B_{1, X} \times B_{1, Y}\right)} \subset \overline{T\left(B_{2, X \times Y}\right)} \subset 4 \overline{T\left(B_{1, X \times Y}\right)} .
$$

Since any closed subset of a compact set is compact, our assertion follows.

$(\mathrm{c} 2) \Rightarrow(\mathrm{c} 3)$ We simply need to notice that

$$
\overline{T\left(B_{r, X \times Y}\right)}=r^{2} \overline{T\left(B_{1, X \times Y}\right)} .
$$

(c3) $\Rightarrow$ (c4) Assuming $0<r_{1} \leq r_{2}$,

$$
\overline{T\left(B_{r_{1}, X \times Y}\right)} \subset \overline{T\left(B_{r_{1}, X} \times B_{r_{2}, Y}\right)} \subset \overline{T\left(B_{r_{1}+r_{2}, X \times Y}\right)} .
$$

Again, since any closed subset of a compact set is compact, the assertion follows.

$(\mathrm{c} 4), \mathrm{c}(6) \Rightarrow(\mathrm{c} 1)$ and $(\mathrm{c} 5) \Rightarrow(\mathrm{c} 2)$ are trivially true.

$(\mathrm{c} 2) \Rightarrow(\mathrm{c} 5)$ and $(\mathrm{c} 3) \Rightarrow(\mathrm{c} 6)$ follow from the definition of boundedness.

(c1) $\Leftrightarrow(\mathrm{c} 7)$ follows from the fact that in any metric space, precompactness is equivalent to sequential compactness.

$(\mathrm{c} 1) \Leftrightarrow(\mathrm{c} 8)$ is similar. Under the additional assumption that $Z$ is Banach, we know that $T(B)$ is precompact if and only if $\overline{T(B)}$ is totally bounded, which is the same as having $T(B)$ totally bounded.

4.2. Proof of Proposition 2. Let $U \subset X \times Y$ be bounded and $\left\{T\left(x_{n}, y_{n}\right)\right\}$ be a sequence in $T(U)$. Let $z_{n}=T\left(x_{n}, y_{n}\right), z_{n}^{\prime}=T_{1}\left(x_{n}, y_{n}\right)$, and $z_{n}^{\prime \prime}=T_{2}\left(x_{n}, y_{n}\right)$ so that $z_{n}=\alpha z_{n}^{\prime}+\beta z_{n}^{\prime \prime}$. Since $T_{1}$ and $T_{2}$ are compact, there exist subsequences $\left\{z_{n_{k}}^{\prime}\right\}$ and $\left\{z_{n_{k_{j}}}\right\}$ that are convergent in $Z$. Let $z_{n_{k_{j}}}=\alpha z_{n_{k_{j}}}^{\prime}+\beta z_{n_{k_{j}}}^{\prime \prime}$. Then $\left\{z_{n_{k_{j}}}\right\}$ is convergent, so $T(U)$ is precompact. The result follows from (c5). 
4.3. Composition with compact bilinear operators. Assume that $T \in \mathcal{B}(X \times$ $Y, Z)$ and $S_{1}: X \rightarrow X, S_{2}: Y \rightarrow Y, S_{3}: Z \rightarrow Z$ are three linear operators. We define the concept of composition between linear and bilinear operators as follows.

Definition 3. Left composition:

$$
S_{3} T: X \times Y \rightarrow Z, S_{3} T(x, y)=S_{3}(T(x, y)) .
$$

Right composition:

$$
T\left(S_{1}, S_{2}\right): X \times Y \rightarrow Z, T\left(S_{1}, S_{2}\right)(x, y)=T\left(S_{1}(x), S_{2}(y)\right) .
$$

We have the following straightforward result.

Proposition 4. If $T \in \mathcal{K}(X \times Y, Z)$ and $S_{1}, S_{2}, S_{3}$ are bounded, then $S_{3} T \in$ $\mathcal{K}(X \times Y, Z)$ and $T\left(S_{1}, S_{2}\right) \in \mathcal{K}(X \times Y, Z)$.

4.4. Some elementary examples. Here are a few more illustrations of the concepts of compactness in bilinear setting. Some of the examples below are modifications of classical ones used in other topics in functional analysis.

Example 1. Let $X=Y=Z=C(0,1)$ be the space of continuous functions on $[0,1]$ now endowed with the supremum norm. Again define $T: X \times Y \rightarrow Z$ by $T(f, g)=f \cdot g$. If we fix $f=1$ (the constant function 1 ), we get that $T_{f}=I d_{X}$ (the identity operator). Since $X$ is infinitely dimensional, by Riesz's theorem we get that $T_{f}$ is not compact. So $T$ is compact in neither the first nor the second variable.

Example 2. To wit, with the notation in the previous example, if $S: X \rightarrow X$ is compact, then $T_{1}(f, g)=S(f) g$ is compact in the first variable but not in the second, while $T_{2}(f, g)=f S(g)$ is compact in the second variable but not the first. Clearly then, neither of the $T_{i}$ 's are separately compact, and thus these $T_{i}$ 's are not compact either.

Example 3. We again let $X=Y=Z=C(0,1)$ be endowed with the supremum norm, but now

$$
T(f, g)(x)=\int_{0}^{x} f(t) g(t) d t .
$$

Clearly, $T$ is a well defined bilinear operator. Let

$$
K=T\left(B_{1} \times B_{1}\right) .
$$

Note that if $h=T(f, g) \in K$, then

$$
\|h\|_{\infty} \leq\|f\|_{\infty}\|g\|_{\infty}<1
$$

that is, $K$ is a bounded set in the supremum norm of $Z$.

Furthermore, if $x, y \in[0,1]$ and $h=T(f, g) \in K$, we have

$$
|h(x)-h(y)|=\left|\int_{x}^{y} f(t) g(t) d t\right| \leq|x-y|,
$$

which proves that $K$ is a uniformly equicontinuous subset of $Z$.

Therefore, using the Arzelà-Ascoli theorem, we conclude that $K$ is precompact in $Z$, and hence $T$ is a bilinear compact operator. 
Example 4. Consider yet again $X=Y=Z=C(0,1)$, but now $X$ and $Y$ are endowed with the $L^{1}$ norm while $Z$ is endowed with the supremum norm. The bilinear operator $T$ is the one defined in Example 3.

Let us fix $f \neq 0 \in X$ and write $K_{f}=T_{f}\left(B_{1}\right)$. Then, if $h=T_{f}(g) \in K_{f}, g \in Y$ (with the modified topology induced by $L^{1}$ ), we have

$$
\|h\|_{\infty} \leq\|f\|_{\infty}\|g\|_{1}<\|f\|_{\infty} ;
$$

that is, $K_{f}$ is a bounded set in the supremum norm of $Z$.

Furthermore, similarly as above, we get that

$$
|h(x)-h(y)| \leq\|f\|_{\infty}|x-y|,
$$

which shows that $K_{f}$ is a uniformly equicontinuous subset of $Z$. Consequently, Arzelà-Ascoli's theorem shows that $K_{f}$ is precompact in $Z$. If $f=0$ (the constant function 0$)$, then $T_{f}\left(B_{1}\right)=\{0\}$, which is obviously precompact. Analogously, $T_{g}\left(B_{1}\right)$ is precompact for all $g \in Y$; hence $T$ is separately compact.

However, consider the sequence $\left(f_{n}\right)$ of continuous functions defined as follows:

$$
f_{n}(t)=\left\{\begin{array}{rll}
4 n^{3} t & \text { if } & 0 \leq t<1 /\left(4 n^{2}\right), \\
n & \text { if } 1 /\left(4 n^{2}\right) \leq t<3 /\left(4 n^{2}\right), \\
-4 n^{3} t+4 n & \text { if } 3 /\left(4 n^{2}\right) \leq t<1 / n^{2}, \\
0 & \text { if } 1 / n^{2} \leq t \leq 1 .
\end{array}\right.
$$

Then $f_{n} \rightarrow 0$ in $X$ (and $Y$ ). Now, for any $x \in(0,1]$, let $N \in \mathbb{N}$ be such that $x>1 / N^{2}$. For all $n>N$, we can write

$$
T\left(f_{n}, f_{n}\right)(x)=\int_{0}^{x} f_{n}^{2}(t) d t=\int_{0}^{1} f_{n}^{2}(t) d t=\frac{2}{3},
$$

which proves that

$$
\left\|T\left(f_{n}, f_{n}\right)\right\|_{\infty} \not \rightarrow 0
$$

or $T$ is not bounded. Consequently, $T$ is not compact.

Example 5. Let $\left(\alpha_{i j k}\right)_{i, j, k=1}^{\infty} \in l^{1}$, that is,

$$
\sum_{i, j, k=1}^{\infty}\left|\alpha_{i j k}\right|<\infty
$$

Define $T: l^{2} \times l^{2} \rightarrow l^{1}$ as follows: for $x=\left(x_{n}\right), y=\left(y_{n}\right), z=\left(z_{n}\right)$,

$$
T(x, y)=z \Leftrightarrow z_{i}=\sum_{j, k=1}^{\infty} \alpha_{i j k} x_{j} y_{k} .
$$

Note that the operator is well defined, since by the Cauchy-Schwarz inequality we have

$$
\sum_{i}\left|z_{i}\right| \leq\left(\sum_{i, j, k}\left|\alpha_{i j k}\right|\right)\|x\|_{l^{2}}\|y\|_{l^{2}} .
$$

Obviously, $T$ is bilinear. Furthermore, if we let $z=\left(z_{n}\right)$ be as before, we can define

$$
T_{n}: l^{2} \times l^{2} \rightarrow l^{1}, T_{n}(x, y)=\left(z_{1}, z_{2}, \ldots, z_{n}, 0,0, \ldots\right) .
$$

Since $T_{n}\left(l^{2} \times l^{2}\right)$ is contained in an $n$-dimensional subspace of $l^{1}$, and since clearly for any bounded subset $U$ of $l^{2} \times l^{2}$ we have that $T_{n}(U)$ is a bounded subset of an 
$n$-dimensional subspace of $l^{1}$, we conclude that necessarily $T_{n}(U)$ is precompact. Thus, $T_{n}$ is a compact bilinear operator. Now, for all $x, y \in B_{1, l^{2}}$, we have

$$
\left\|T_{n}(x, y)-T(x, y)\right\|=\left\|\left(0, \ldots, 0, z_{n+1}, \ldots\right)\right\|_{l^{1}} \leq \sum_{i=n+1}^{\infty} \sum_{j, k=1}^{\infty}\left|\alpha_{i j k}\right|,
$$

which in turn implies that $\left\|T_{n}-T\right\| \rightarrow 0$ as $n \rightarrow \infty$. By Proposition 3 we conclude that $T$ is a compact bilinear operator.

Example 6. Consider a kernel $K \in C\left([0,1]^{3}\right)$, and define $T: L^{2}(0,1) \times L^{2}(0,1) \rightarrow$ $C(0,1)$ by

$$
T(f, g)(s)=\int_{0}^{1} \int_{0}^{1} K(s, t, u) f(t) g(u) d t d u .
$$

Then, clearly, $T$ is well defined and bilinear. Moreover, the family $\{T(f, g): f, g \in$ $\left.B_{1, L^{2}}\right\}$ is equicontinuous, since

$$
\left|T(f, g)\left(s_{1}\right)-T(f, g)\left(s_{2}\right)\right| \leq \max _{t, u \in[0,1]}\left|K\left(s_{1}, t, u\right)-K\left(s_{2}, t, u\right)\right| .
$$

Moreover, for all $s \in[0,1]$ and for all $f, g \in B_{1}$, we have

$$
|T(f, g)(s)| \leq M=\max _{s, t, v \in[0,1]}|K(s, t, u)| .
$$

In other words, $T\left(B_{1} \times B_{1}\right)$ is equibounded and equicontinuous, that is, precompact. This proves that $T$ is compact.

Example 7. Finally, let $K \in L^{2}\left((0,1)^{3}\right)$ and define $T: L^{2}(0,1) \times L^{2}(0,1) \rightarrow$ $L^{1}(0,1)$ by

$$
T(f, g)(s)=\int_{0}^{1} \int_{0}^{1} K(s, t, u) f(t) g(u) d t d u .
$$

Let $K_{n} \in C\left([0,1]^{3}\right)$ be such that $K_{n} \rightarrow K$ in the $L^{2}$ norm and define the operator $T_{n}$ as in Example 6 , with $K$ replaced by the kernel $K_{n}$. By Cauchy-Schwarz we immediately get that, for all $f, g \in B_{1, L^{2}}$,

$$
\left\|T_{n}(f, g)-T(f, g)\right\|_{L^{1}} \leq\left\|K_{n}-K\right\|_{L^{2}} \rightarrow 0
$$

as $n \rightarrow \infty$. We appeal again to Proposition 3 and conclude that, since $T_{n}$ are compact (see Example 6), $T$ must be compact as well.

\section{REFERENCES}

[1] G. Bourdaud, Remarques sur certains sous-espaces de $B M O\left(R^{n}\right)$ et de bmo $\left(R^{n}\right)$, Ann. Inst. Fourier (Grenoble) 52 (2002), 1187-1218. MR.1927078(2003f:42033)

[2] G. Bourdaud, M. Lanza de Cristoforis, and W. Sickel, Functional calculus on BMO and related spaces, J. Func. Anal. 189 (2002), 515-538. MR1892179 (2003c:47059)

[3] A.P. Calderón, Intermediate spaces and interpolation, the complex method, Studia Math. 24 (1964), 113-190. MR0167830 (29:5097)

[4] R. Coifman, R. Rochberg, and G. Weiss, Factorization theorems for Hardy spaces in several variables, Ann. of Math. 103 (1976), 611-635. MR0412721(54:843)

[5] R. Coifman and G. Weiss, Extension of Hardy spaces and their use in analysis, Bull. Amer. Math. Soc. 83 (1977), 569-645. MR0447954(56:6264)

[6] D.L. Fernandez and E.B. da Silva, Interpolation of bilinear operators and compactness, Nonlinear Analysis 73 (2010), 526-537. MR2650835 (2011h:46036)

[7] L. Grafakos and R.H. Torres, Multilinear Calderón-Zygmund theory, Adv. Math. 165 (2002), 124-164. MR 1880324 (2002j:42029) 
[8] L. Grafakos and R.H. Torres, Maximal operator and weighted norm inequalities for multilinear singular integrals, Indiana Univ. Math. J. 51 (2002), no. 5, 1261-1276. MR.1947875 (2003j:42020)

[9] A. Lerner, S. Ombrosi, C. Pérez, R. Torres, and R. Trujillo-González, New maximal functions and multiple weights for the multilinear Calderón-Zygmund theory, Adv. Math. 220 (4) (2009), 1222-1264. MR2483720 (2010f:42024)

[10] U. Neri, Fractional integration on the space $H^{1}$ and its dual, Studia Math. 53 (1975), 175-189. MR 0388074 (52:8911)

[11] C. Pérez, G. Pradolini, R.H. Torres, and R. Trujillo-González, End-points estimates for iterated commutators of multilinear singular integrals, preprint, arXiv:1004.4976v1 (April 2010), arXiv:1004.4976v2 (March 2011).

[12] C. Pérez and R.H. Torres, Sharp maximal function estimates for multilinear singular integrals, Contemp. Math. 320 (2003), 323-331. MR1979948 (2004e:42022)

[13] W. Rudin, Functional Analysis, McGraw-Hill, 1973. MR0365062 (51:1315)

[14] D. Sarason, Functions of vanishing mean oscillation, Trans. Amer. Math. Soc. 207 (1975), 391-405. MR0377518 (51:13690)

[15] M. Schechter, Principles of Functional Analysis, Graduate Studies in Mathematics 36, American Mathematical Society, 2002. MR1861991 (2002j:46001)

[16] E.M. Stein, Harmonic Analysis: Real-Variable Methods, Orthogonality, and Oscillatory Integrals, Princeton Univ. Press (1993). MR1232192 (95c:42002)

[17] L. Tang, Weighted estimates for vector-valued commutators of multilinear operators, Proc. Roy. Soc. Edinburgh Sect. A 138 (2008), 897-922. MR2436447(2010a:42055)

[18] A. Uchiyama, On the compactness of operators of Hankel type, Tôhoku Math. J. (2) 30 (1978), no. 1, 163-171. MR0467384 (57:7243)

[19] K. Yosida, Functional Analysis, Springer-Verlag, Berlin (1995). MR1336382 (96a:46001)

Department of Mathematics, Western Washington University, 516 High Street, BelLINGHAM, WASHINGTON 98225

E-mail address: arpad.benyi@wwu.edu

Department of Mathematics, University of Kansas, Lawrence, Kansas 66045

E-mail address: torres@math.ku.edu 